\title{
Impacto de la COVID-19 en la práctica deportiva de personas participantes en eventos deportivos de carrera a pie y ciclismo en España y Portugal \\ The impact of COVID-19 on physical activity on people who participate on running and cycling sporting events people in Spain and Portugal \\ *Jordi Segui-Urbaneja, ** Rui Pedro Julião, **Ricardo Manuel Nogueira Mendes, *Víctor Dorado, *Estela I Farías- \\ Torbidoni \\ *Universidad de Lleida (España), **Universidad Nova de Lisboa (Portugal)
}

Resumen. Es objeto de este artículo analizar el efecto de la COVID-19 en la práctica deportiva de personas participantes en eventos deportivos de carrera a pie y ciclismo en España y Portugal. Se consideró la situación de práctica deportiva en España y Portugal previa a la irrupción de la COVID-19, como ambos Gobiernos legislan para proteger a los ciudadanos de la pandemia y se analizan las consecuencias de éstas. Se realizó una investigación descriptiva mediante el uso de cuestionario $(\mathrm{N}=2696)$ y análisis estadístico descriptivo según características y distribución (prueba de la normalidad) de las diferentes variables: frecuencias, valores medios, máximas y mínimas, mediana e IQR. De los resultados obtenidos se puede destacar: a) la casi plena coincidencia entre estados del perfil genérico de los practicantes; el orden por porcentaje de las variables, e incluso, los porcentajes de cada variable para cuatro de las cinco dimensiones analizadas; b) la dimensión que más difiere fue como se adaptaron durante la fase de confinamiento las personas practicantes en España y Portugal, y c) que en todo momento las personas encuestadas percibieron que las adaptaciones serían pasajeras y era cuestión de algunos meses que se volviera a la normalidad. Estos datos permiten entender el efecto que la pandemia de la COVID-19 tuvo en la práctica deportiva y de participantes de eventos deportivos en el medio natural en España y Portugal.

Palabras clave: COVID-19, práctica deportiva, carrera a pie, ciclismo.

Abstract. The aim of this article is to analyze the impact of COVID-19 on physical activity on people who participate on running and cycling sporting events people in Spain and Portugal. It was considered The situation of sports practice in Spain and Portugal prior to the outbreak of COVID-19, as both governments legislate to protect citizens from the pandemic and it was analyzed the consequences of these acts. A descriptive investigation was carried out using a questionnaire $(\mathrm{N}=2696)$ and descriptive statistical analysis according to characteristics and distribution (test of normality) of different variables: frequencies, average, maximum and minimum values, median and IQR. From the results, the following can be highlighted: a) almost complete coincidence between both countries, of the generic profile of the practitioners; the order, by percentage, of the variables, and even the percentages of each variable for four of the five dimensions analyzed; b) the dimension that differs the most is how practitioners in Spain and Portugal adapted during the confinement time, and c) at all times the respondents perceived that the adaptations would be temporary and it was a matter of a few months before they returned to the normality. These data allow to understand the effect of COVID-19 pandemic had on physical activity on people who participate on sporting events in the natural environment in Spain and Portugal.

Keywords: COVID-19, sports practice, running, cycling.

\section{Introducción}

El artículo 43.3 de la Constitución española de 1978 y el artículo 79 de la Constitución portuguesa de 1976 reconocen el deporte y la actividad física como derechos para su ciudadanía. Por ello, tanto el estado español como el estado portugués, entienden que el deporte y la actividad física deben ser fomentadas por las respectivas administraciones públicas en sus respectivos territorios.

El 11 de marzo de 2020, la Organización Mundial de la Salud, por efecto de la COVID-19, elevó la situación de emergencia de salud pública a pandemia internacional. Ante la rapidez en la evolución de los hechos, a escala nacional e internacional, los diferentes estados adoptaron medidas inmediatas de una severidad hasta el momento nunca vistas en muchos territorios. Para hacer frente a la crisis sanitaria tanto el estado español como el portugués respondieron con la publicación de: a) España: el Real Decreto 463/2020, de 14 de marzo, por el que se declara el estado de alarma para la gestión de la situación de crisis sanitaria ocasionada por la COVID-19; y b) Portugal: el Decreto de la Presidencia de la

Fecha recepción: 05-08-20. Fecha de aceptación: 09-09-20

Jordi Segui-Urbaneja

jsegui@inefc.es
República nº14-A/2020, de 18 de marzo, en que se declara el estado de emergencia, con fundamento a una verificación de una situación de calamidad pública.

Ambos gobiernos legislaron de urgencia para proteger la salud y seguridad de la ciudadanía, contener la progresión de la enfermedad y reforzar el sistema de salud público. Las medidas temporales de carácter extraordinario, declaración del estado de alarma -España- y el estado de alarma y situación de calamidad -Portugal-, incidieron de manera directa en la libre circulación de las personas (artículo 7) y en la contención en el ámbito de las actividades recreativas y equipamientos culturales y otros adicionales (artículo 10), en el caso de España; y el confinamiento obligatorio (artículo 2), instalaciones y establecimientos encerrados (artículo 5), actividad física y deportiva (artículo 16) y eventos (artículo 18) en el caso de Portugal.

Así, ambos territorios, declararon el estado de alarma, pero con algunas diferencias, tanto en su devenir como en la repercusión sobre la ciudadanía. La gestión del estado de alarma difirió entre Gobiernos; mientras que en el territorio español el Congreso prorrogó el estado de alarma hasta en seis ocasiones (del 14 de marzo hasta el 21 de junio de 2020); en el territorio portugués el estado de alarma se prorrogó en dos ocasiones (del 19 de marzo hasta el 2 de mayo), siendo substituido por la situación de calamidad en el ámbito de la pandemia de la enfermedad COVID-19, definido en la Reso- 
lución Consejo de Ministros n³3-A/2020, 30 de abril de 2020 (Diário da República ${ }^{\circ} 85 / 2020,3^{\circ}$ suplemento, serie I de 202004-30), con medidas menos restrictivas.

La implicación de las medidas legislativas también fueron diferentes, mientras que en España quedaba restringida la circulación por vías o espacios de uso público para determinadas actividades, la práctica deportiva quedaba excluida (no se pudo realizar práctica deportiva en vías o espacios de uso público hasta la segunda medida de la fase 0 del plan de desescalada -2 de mayo de 2020 - cuando se permitió las salidas para pasear y hacer deporte individual sin salir del propio municipio y en determinadas franjas horarias); en Portugal no se produjo tal restricción y desde el primer día de la declaración del estado de emergencia fue una recomendación, permitiendo así la práctica deportiva en vías o espacios de uso público bajo algunas medidas de protección. Así, mientras que en España hubo un periodo de 20 días con total prohibición para la práctica deportiva en vías o espacios públicos en Portugal nunca se llegó a prohibir.

En este contexto se justifica la necesidad de analizar si la restricción (prohibición en España y recomendación en Portugal) de circulación, reunión y utilización de espacios públicos, tuvo incidencia en la práctica deportiva de la ciudadanía española y portuguesa y, en caso afirmativo, como varió dicha práctica.

\section{La práctica deportiva en España y Portugal antes de}

\section{Ia COVID-19}

El eurobarómetro permite conocer los hábitos deportivos de la población europea. A partir de éstos se dispone de información en relación al porcentaje de población que práctica deporte o actividad física, la intensidad de la práctica, el lugar de práctica y la motivación para practicar. El último eurobarómetro publicado, marzo de 2018 (European Union, 2018), destaca que: a) el 7\% de la población europea practica algún deporte de forma regular (14\% en España y 5\% en Portugal) y el $33 \%$ lo hace con alguna regularidad (29\% en España y 21\% en Portugal); b) atendiendo a la intensidad de la actividad física, el estudio diferencia entre vigoroso, moderado, caminar o estar sentado. Vigoroso correspondería a entrenamientos típicos de personas que se preparan para algún evento deportivo. Así en relación a la intensidad vigorosa, el 15\% lo realiza entre 4 y 7 días (12\% en España y $7 \%$ en Portugal), el $27 \%$ entre 1 y 3 días (21\% en España y $13 \%$ en Portugal) y el $58 \%$ no lo practica nunca (67\% en España y $79 \%$ en Portugal; c) respecto al lugar de práctica, destaca que el $40 \%$ de las personas practicantes de actividad física en la Unión Europea realiza deporte en parques y espacios al aire libre (53\% en España y $43 \%$ en Portugal) y el $32 \%$ lo hace en casa ( $16 \%$ en España y $17 \%$ en Portugal); y d) el estudio también expone que las dos principales motivaciones para la realización de actividad física son la mejora de la salud para el $54 \%$ de la población (59\% en España y 51\% en Portugal) y la forma física para el 47\% (38\% en España y 36\% en Portugal).

Como datos complementarios, en el caso de España, resultan de especial importancia los obtenidos en la última encuesta de Hábitos Deportivos (CSD, 2017). De ella y atendiendo al lugar donde suele practicar deporte, se observa que el $45,9 \%$ de personas practicantes lo hacen en el aire libre, siendo este el principal espacio de práctica. Si se refiere a las personas que practican deporte según modalidades deportivas más frecuentes, destaca la práctica del ciclismo (38,7\% en el último año y $22,2 \%$ semanalmente), senderismo y montañismo (31,9\% en el último año y $10,5 \%$ semanalmente) y carrera a pie (30,4\% en el último año y $23,0 \%$ semanalmente) siendo la primera, tercera y cuarta modalidad más practicada en el último año y semanalmente. En Portugal, no se disponen de estudios públicos específicos que permitan conocer los hábitos deportivos de su población.

El conjunto de datos disponibles, describen un contexto en relación a la población que practica deporte o actividad física junto a algunas características de dicha práctica que supone el punto de partida para el análisis del presente estudio.

A partir del contexto anteriormente descrito en el que, en el territorio español y portugués, se declaró el estado de alarma y calamidad y se restringió, en menor o mayor medida, la libre circulación de personas, la limitación de actividades recreativas, actividad física y deportiva, la utilización de instalaciones y establecimientos cerrados, y eventos. Y, teniendo en cuenta los contextos territoriales de práctica deportiva en cuanto a porcentaje de población que practica y la intensidad con la que lo hace, los lugares de práctica, las modalidades más practicadas y la motivación para hacerlo; es que la presente investigación realiza un análisis para saber cómo ha afectado (o no) el estado de alarma y situación de calamidad en la práctica deportiva en la población participante en eventos deportivos de carrera a pie y ciclismo española y portuguesa $y$, en caso de producirse una modificación, en qué ha consistido y si está ha sido igual para ambos territorios.

Para dar respuesta a dicha inquietud se han planteado tres objetivos: 1) identificar los hábitos deportivos antes de la declaración del estado de alarma en España y el estado de calamidad en Portugal (perspectiva previa); 2) conocer la adaptación de la población encuestada a ambos contextos (perspectiva durante); y 3) saber como cree, la población encuestada, que será la práctica deportiva una vez se supriman las restricciones (perspectiva posterior).

\section{Metodología}

El método utilizado para realizar este estudio se basó en la aplicación de una encuesta en formato online. La misma fue implementada utilizando la plataforma Survey 123 para ArcGIS en dos idiomas (castellano para España y portugués para Portugal). Para la implementación de las encuestas se contó, selección de la muestra por conveniencia, con la colaboración de tres empresas especializadas en la calendarización, cronometraje y organización de eventos deportivos en ambos países: Timing Lap e Iter5 en el caso de España y Lap2Go en el caso de Portugal. En la tabla 1 se expone el detalle del número de encuestas finalmente recogido en las diferentes plataformas una vez eliminados los ca-

\begin{tabular}{|c|c|c|c|c|c|}
\hline $\begin{array}{c}\text { Propietario } \\
\text { base de datos }\end{array}$ & $\begin{array}{c}\text { Fecha de } \\
\text { implementación }\end{array}$ & $\begin{array}{c}\text { Emails } \\
\text { remitidos }\end{array}$ & $\begin{array}{c}\text { Emails } \\
\text { visualizados }\end{array}$ & $\begin{array}{c}\text { Tasa de } \\
\text { respuesta }\end{array}$ & $\begin{array}{l}\text { Encuestas } \\
\text { obtenidas }\end{array}$ \\
\hline Lap2Go & 30 de abril & 48384 & 20493 & $11.36 \%$ & 2245 \\
\hline Timing Lap & 3 de mayo & 18310 & 6035 & $5.13 \%$ & 309 \\
\hline ITER5 & 5 de mayo & Sin datos & Sin datos & Sin datos & 142 \\
\hline Total & & & & & 2696 \\
\hline
\end{tabular}


sos pertenecientes a personas encuestadas con residencia habitual fuera de España y Portugal (72 casos) y menores de 16 años (13 casos.).

El diseño de encuesta tuvo en consideración cinco grandes dimensiones: perfil sociológico (ej. edad, género, dedicación laboral, etc.); perfil deportivo (práctica deporte individual, modalidad, etc.); práctica deportiva previa al confinamiento (ej. grupo entrenamiento, entrenador, uso de gimnasio, etc.); práctica deportiva durante el confinamiento (ej. cambios en frecuencia y duración de práctica, cambios del lugar de práctica, incorporación de nuevos equipamientos y/o complementos, etc.); y práctica deportiva posterior al confinamiento (ej. cambios en la frecuencia y duración, cambios del lugar de práctica, incorporación de nuevos equipamientos y/o complementos, etc.). En la tabla 2 de puede apreciar en detalle las dimensiones y variables consideradas en el modelo de encuesta.

La participación en el estudio fue voluntaria y cumplió con todos los requerimientos derivados de las leyes de protección de datos personales y los derechos digitales de ambos estados. Ley Orgánica 3/2018, de 5 de diciembre (España) y de Ley n. ${ }^{\circ}$ 58/2019, de 8 de agosto (Portugal).

Los datos obtenidos fueron transformados y codificados utilizando el SPSS, versión 25.0 (IBM Corp., 2017). El análisis de datos se basó en la aplicación de análisis estadístico descriptivo según características y distribución (prueba de la normalidad) de las diferentes variables: frecuencias, valores medios, máximas y mínimas, mediana, IQR.

Tabla 2

\begin{tabular}{|c|c|}
\hline Dimensiones & Variables \\
\hline Perfil sociológico & $\begin{array}{l}\text { Edad, género, dedicación laboral, tipo de contrato, cambio } \\
\text { dedicación laboral durante el confinamiento }\end{array}$ \\
\hline Perfil deportivo & Practica deporte individual, modalidad, antigüedad, revisión médica \\
\hline $\begin{array}{l}\text { Práctica deportiva previa } \\
\text { al confinamiento }\end{array}$ & $\begin{array}{l}\text { Formato de práctica, entrenador, uso de gimnasio, uso de } \\
\text { app/software, número de sesiones semanales, participación en } \\
\text { eventos deportivos } 2019\end{array}$ \\
\hline $\begin{array}{l}\text { Práctica deportiva durante } \\
\text { el confinamiento }\end{array}$ & $\begin{array}{l}\text { Cambio en la actividad regular, número de sesiones semanales, } \\
\text { cambios de lugar de práctica, percepción cambio de técnica, } \\
\text { percepción cambio rendimiento fisico, inclusión de } \\
\text { complementos/equipamiento, adquisición de equipamientos, } \\
\text { incorporación uso de nuevos app/software, incorporación entrenador }\end{array}$ \\
\hline $\begin{array}{l}\text { Práctica deportiva } \\
\text { posterior al confinamiento }\end{array}$ & $\begin{array}{l}\text { Abandono práctica modalidad deportiva principal, cambio en la } \\
\text { forma de practica, expectativa retorno a práctica habitual y } \\
\text { participación en eventos deportivos, expectativa cambios en el } \\
\text { formato de los eventos deportivos, tiempo para retorno a la práctica } \\
\text { deportiva habitual }\end{array}$ \\
\hline
\end{tabular}

\section{Resultados}

A continuación, se presentan la concreción de los resultados según las dimensiones analizadas.

\section{Perfil sociodemográfico}

La mayoría de las personas que respondieron el cuestionario se caracterizan por tener una edad comprendida entre los 36 y los 45 años (40.6\% España y 43.1\% Portugal), ser de género masculino (78.2\% España y $71.5 \%$ Portugal); empleado por cuenta ajena (53.7\% España y 58.8\% Portugal), la mayor parte de los cuales con un tipo de contrato a tiempo completo ( $72.1 \%$ España y $76.9 \%$ Portugal) y no sufrió cambio laboral alguno durante el periodo de confinamiento (38.9\% España y $42.0 \%$ Portugal). Así se identifica un perfil de persona adulta y con un perfil laboralmente consolidado que coincide con los mismos valores en ambos estados. Tabla 3 .

Conviene destacar la identificación de un perfil mayoritario es una persona adulta, social y familiarmente estable (edad entre 36 y 45 años) con una carrera profesional conso-

\begin{tabular}{|c|c|c|c|}
\hline Variables & $\begin{array}{c}\text { España (\%) } \\
n=451\end{array}$ & $\begin{array}{c}\text { Portugal (\%) } \\
n=2245\end{array}$ & $\begin{array}{c}\text { Total muestra }(\%) \\
\mathrm{N}=2696\end{array}$ \\
\hline \multicolumn{4}{|l|}{ Edad } \\
\hline $16-25$ años & 3.5 & 3.9 & 3.9 \\
\hline $26-35$ años & 15.7 & 20.0 & 19.3 \\
\hline $36-45$ años & 40.6 & 43.1 & 42.7 \\
\hline $46-55$ años & 29.9 & 25.3 & 26.0 \\
\hline $56-65$ años & 8.4 & 6.5 & 6.8 \\
\hline Más de 65 años & 1.8 & 1.3 & 1.4 \\
\hline Edad (años). Media (SD). & $43.46(9.61)$ & $41.93(9.30)$ & $42.19(9.4)$ \\
\hline Edad (años). $\mathrm{M}_{\mathrm{e}}[\mathrm{IQR}]$. & $43.00[37.00,50.00]$ & $42.00[36.00,48.00]$ & $42.00[36.00,48.00]$ \\
\hline \multicolumn{4}{|l|}{ Género } \\
\hline Masculino & 78.2 & 71.5 & 72.6 \\
\hline Femenino & 21.6 & 28.4 & 27.3 \\
\hline No binario & 0.2 & 0.1 & 0.1 \\
\hline \multicolumn{4}{|l|}{ Dedicación laboral } \\
\hline Estudiante & 4.5 & 2.4 & 2.7 \\
\hline Empleado por cuenta ajena & 53.7 & 58.8 & 58.0 \\
\hline Funcionario & 22.3 & 20.7 & 21.0 \\
\hline Autónomo, empresario & 10.2 & 12.5 & 12.1 \\
\hline Jubilado & 3.6 & 2.0 & 2.2 \\
\hline Parado/desocupado & 5.6 & 3.1 & 3.5 \\
\hline Otras opciones & 0.2 & 0.5 & 0.5 \\
\hline \multicolumn{4}{|l|}{ Tipo de contrato } \\
\hline A tiempo completo & 72.1 & 76.9 & 76.1 \\
\hline A tiempo parcial & 4.1 & 1.0 & 1.6 \\
\hline Autónomo/empresario & 8.4 & 11.8 & 11.2 \\
\hline Otras opciones & 1.6 & 2.8 & 2.6 \\
\hline No corresponde & 13.8 & 7.6 & 8.6 \\
\hline \multirow{2}{*}{\multicolumn{4}{|c|}{ Cambio periodo de confinamiento }} \\
\hline & & & \\
\hline Teletrabajo & 38.9 & Sin alteración & 41.5 \\
\hline ERTE & 29.8 & 31.6 & 31.3 \\
\hline Desempleo & 8.7 & 12.9 & 12.0 \\
\hline & 1.8 & 1.6 & 1.7 \\
\hline \multirow[t]{2}{*}{ No corresponde } & 7.8 & 4.5 & 4.9 \\
\hline & 13.6 & 7.5 & 8.5 \\
\hline
\end{tabular}

lidada (el 76\% en España y el 79.5\% en Portugal es trabajador por cuenta ajena o funcionario y el $72 \%$ en España y el $76 \%$ en Portugal trabajan a tiempo completo) que no presentó grandes variaciones durante el periodo de confinamiento. Solo el $8.7 \%$ España y $12.9 \%$ en Portugal han sufrido ERTE y el $1.8 \%$ en España y el 1.6\% en Portugal han desempleado. Puestos laborales consagrados.

Un análisis específico merece la variable de género, así destaca el alta, aunque aún lejos de la paridad, participación de las féminas ( $21 \%$ en España y $28 \%$ en Portugal), más aún teniendo en cuenta que el perfil de sujeto que ha tenido acceso al cuestionario es una persona que participa en competiciones deportivas.

\section{Perfil deportivo}

La persona tipo que contestó el cuestionario se caracteriza por practicar deporte individual (96.5\% España y 95.1\% Portugal), en las modalidades de carrera (65.9\% España y $72.3 \%$ Portugal) y ciclismo (17.7\% España y $10.5 \%$ Portugal $)$ ambas en sus dos modalidades de asfalto o montaña, llevar alrededor de cinco años practicando el deporte principal (63.6\% España y $41.2 \%$ Portugal), y no pasar revisión médi-

\begin{tabular}{|c|c|c|c|}
\hline Variables & $\begin{array}{c}\text { España (\%) } \\
n=451\end{array}$ & $\begin{array}{c}\begin{array}{c}\text { Portugal }(\%) \\
n=2245\end{array} \\
\end{array}$ & $\begin{array}{c}\text { Total muestra (\%) } \\
\mathrm{N}=2696 \\
\end{array}$ \\
\hline \multicolumn{4}{|l|}{ Práctica deporte individual } \\
\hline Sí & 96.5 & 95.1 & 95.3 \\
\hline No & 3.5 & 4.9 & 4.7 \\
\hline \multicolumn{4}{|l|}{ Modalidad deportiva principal } \\
\hline Carreras por asfalto & 43.7 & 39.0 & 39.8 \\
\hline Trail running & 22.2 & 33.3 & 31.4 \\
\hline Mountain bike & 13.0 & 6.2 & 7.3 \\
\hline Ciclismo de carretera & 4.7 & 4.3 & 4.3 \\
\hline Triatlón & 7.6 & 2.5 & 3.4 \\
\hline Otros & 5.2 & 9.7 & 9.1 \\
\hline No corresponde & 3.6 & 5.0 & 4.7 \\
\hline \multicolumn{4}{|l|}{ Antigüedad modalidad principal } \\
\hline Menos de un año & 1.6 & 4.0 & 3.6 \\
\hline Entre 1 y 3 años & 12.9 & 25.0 & 23.0 \\
\hline Entre 4 y 5 años & 18.3 & 24.8 & 23.7 \\
\hline Más de 5 años & 63.6 & 41.2 & 45.0 \\
\hline No corresponde & 3.6 & 5.0 & 4.8 \\
\hline \multirow{2}{*}{$\begin{array}{l}\text { Antigüedad (años). Media (SD). } \\
\text { Antigüedad (años). } M_{e}[\text { IQR }] .\end{array}$} & $11.11(9.60)$ & $7.71(7.47)$ & $8.30(7.99)$ \\
\hline & $8.00[5.00 ; 13.00]$ & $5.00[3.00,9.00]$ & $5.00[3.00,10.00]$ \\
\hline \multicolumn{4}{|l|}{ Revisión médica } \\
\hline Sí & 34.1 & 43.4 & 41.8 \\
\hline No & 65.9 & 56.6 & 58.2 \\
\hline
\end{tabular}


ca $(65.9 \%$ España y $56.6 \%$ Portugal). Se identifica un perfil de practicante de deportes individuales, principalmente ciclismo y carrera, consolidado que no realiza revisiones médicas. Todas las variables coinciden tanto en España como en Portugal. Tabla 4.

Destacar una mayor presencia en España de practicantes de triatlón y bicicleta mientras que en Portugal de practicantes de carrera a pie. Respecto a la antigüedad, existe una coincidencia en el orden de la variable, pero no en porcentaje. Así España presenta deportistas «más veteranos» $(63.6 \%$ tienen una antigüedad de práctica mayor a cinco años, mientras que en Portugal representan el 41.2\%), y en Portugal hay un margen más estrecho entre las variables de inicio (entre 1 y 3 años y 4 y 5 años).

Finalmente parece que la persona practicante en Portugal está más concienciado en realizar revisiones médicas (el $43.4 \%$ de practicantes se somete a revisión médica en Portugal mientras que en España representa el 34.1\%).

\section{Práctica deportiva previa al confinamiento}

En relación a los hábitos de práctica deportiva previos al confinamiento, las personas encuestadas declararon tener por hábito de práctica deportiva el formato individual (49.2\% España y $46.2 \%$ Portugal) y entrenar sin entrenador (65.6\% España y $70.3 \%$ Portugal). Respecto al uso, o no, de gimnasio, destaca la escasa diferencia entre los que no lo utiliza y los que si ( $+4.6 \%$ España y $8.6 \%$ Portugal) siendo mayoritario el no uso de gimnasio. Sobre utilizar, o no, app o software durante el entrenamiento, coincide el no uso de estos recur$\operatorname{sos}(56.4 \%$ España y $51.1 \%$ Portugal). Finalmente, respecto al número de sesiones semanales y participación en eventos deportivos, la mayor parte de las personas encuestadas manifestaron realizar 2 o más sesiones semanales (94.4\% España y $94.3 \%$ Portugal) y haber participado en eventos deportivos en el año 2019 (87.3\% España y 89.7\% Portugal) con una media de participación de eventos en el año 2019 muy parecida (9.22 España y 1.48 Portugal). Se identifica unas personas practicantes con hábitos deportivos similares, practicantes individuales, autodidactas (sin entrenador ni App i software) y dedicados (número de sesiones semanales superior a 4 y número medio de participación en eventos deportivos durante el 2019 superior a 9) Tabla 5.

\begin{tabular}{|c|c|c|c|}
\hline Variables & $\begin{array}{c}\text { España (\%) } \\
\mathrm{n}=451\end{array}$ & $\begin{array}{c}\text { Portugal (\%) } \\
\mathrm{n}=2245\end{array}$ & $\begin{array}{c}\text { Total muestra (\%) } \\
\mathrm{N}=2696\end{array}$ \\
\hline \multicolumn{4}{|l|}{ Formato (grupo) } \\
\hline Individual & 49.2 & 46.2 & 46.7 \\
\hline Grupo de amigos & 24.7 & 32.3 & 31.0 \\
\hline Grupo amigos (Redes sociales) & 1.5 & 4.4 & 3.9 \\
\hline Entidades deportivas & 22.1 & 15.8 & 16.9 \\
\hline Otros & 2.5 & 1.3 & 1.5 \\
\hline \multicolumn{4}{|l|}{ Guía con entrenador } \\
\hline Sí & 34.4 & 29.7 & 30.5 \\
\hline No & 65.6 & 70.3 & 69.5 \\
\hline \multicolumn{4}{|l|}{ Uso de gimnasio } \\
\hline $\mathrm{Si}$ & 47.7 & 47.2 & 47.3 \\
\hline No & 52.3 & 52.8 & 52.7 \\
\hline \multicolumn{4}{|l|}{ Uso de Apps/Software } \\
\hline Sí & 43.6 & 48.9 & 48.0 \\
\hline No & 56.4 & 51.1 & 52.0 \\
\hline \multicolumn{4}{|l|}{ Número de sesiones semanales } \\
\hline Menos de 1 sesión & 0.9 & 1.0 & 1.0 \\
\hline 1 sesión & 4.7 & 4.7 & 4.7 \\
\hline Entre 2 y 3 sesiones & 42.1 & 47.5 & 46.6 \\
\hline Entre 4 y 6 sesiones & 47.8 & 41.7 & 42.7 \\
\hline Más de 7 sesiones & 4.5 & 5.1 & 5.0 \\
\hline \multicolumn{4}{|l|}{ Participación eventos deportivos 2019} \\
\hline Sí & 87.3 & 89.7 & 89.3 \\
\hline No & 12.7 & 10.3 & 10.7 \\
\hline Eventos 2019 (número). Media (SD). & $9.22(9.21)$ & $10.48(9.09)$ & $10.28(9.12)$ \\
\hline Eventos 2019 (número). M [IOR]. & $6.00[3.00,12.00]$ & $8.00[4.00,14.00]$ & $8.00[4.00,13.00]$ \\
\hline
\end{tabular}

Entrando en más detalle, los datos muestran la presencia de una mayor práctica deportiva vinculada a entidades deportivas en el caso de España (22.1\% en España y el 15.8\% en Portugal) mientras que en Portugal se realiza en grupo de amigos (32.3\% en Portugal y el $24.7 \%$ en España); como así también una mayor frecuencia de práctica. Esto es, a pesar que los resultados de número de sesiones realizadas presentan una cierta coincidencia de valores en los extremos, no así el número de sesiones intermedias. Así el/la practicante Español/a realiza más sesiones (entre 4 y 6, 47.8\% en España y el $41.7 \%$ en Portugal) y Portugal realiza menos (entre 2 y 3 , $42.1 \%$ en España y el $47.5 \%$ en Portugal).

\section{Práctica deportiva durante el confinamiento}

Respecto a la adaptación de la práctica deportiva durante la fase de confinamiento, las personas encuestadas respondieron que durante la fase de confinamiento sufrieron una disminución de la frecuencia o duración de la actividad física (56.2\% España y $61.8 \%$ Portugal), realizaron dos o más sesiones semanales (91.2\% España y 77.7\% Portugal) suponiendo un cambio a mayor práctica indoor (58.7\%) en el caso de España y alternativamente según la circunstancia (33.8\%) en Portugal. En cuanto a la percepción sobre el rendimiento, vieron ligeramente afectado el cambio de técnica $(57.7 \%$ España y $56.8 \%$ Portugal $)$ y rendimiento físico $(57.3 \%$ España y $58.0 \%$ Portugal).

\begin{tabular}{|c|c|c|c|}
\hline Variables & $\begin{array}{c}\text { España (\%) } \\
\mathrm{n}=451\end{array}$ & $\begin{array}{c}\text { Portugal }(\%) \\
\mathrm{n}=2245\end{array}$ & $\begin{array}{c}\text { Total muestra (\%) } \\
\mathrm{N}=2696\end{array}$ \\
\hline \multicolumn{4}{|l|}{ Cambios en la actividad física regular } \\
\hline Sin cambios relevantes & 15.6 & 15.5 & 15.5 \\
\hline Aumento de frecuencia/duración & 12.2 & 8.6 & 9.2 \\
\hline Disminución de frecuencia/duración & 56.2 & 61.8 & 60.8 \\
\hline Abandonó la actividad física regular & 16.0 & 14.2 & 14.5 \\
\hline \multicolumn{4}{|l|}{ Número de sesiones semanales } \\
\hline Menos de 1 sesión & 2.8 & 4.5 & 4.2 \\
\hline 1 sesión & 6.0 & 17.7 & 15.7 \\
\hline Entre 2 y 3 sesiones & 33.3 & 50.5 & 47.7 \\
\hline Entre 4 y 6 sesiones & 41.0 & 20.3 & 23.8 \\
\hline Más de 7 sesiones & 16.9 & 6.9 & 8.6 \\
\hline \multicolumn{4}{|l|}{ Cambios de lugar de práctica } \\
\hline Sin cambios relevantes & 4.9 & 24.2 & 21.0 \\
\hline Cambio a una mayor práctica indoor & 58.7 & 22.0 & 28.0 \\
\hline Cambio a una mayor práctica outdoor & 4.5 & 5.7 & 5.5 \\
\hline Alternancia según circunstancia & 15.7 & 33.8 & 30.8 \\
\hline No corresponde & 16.2 & 14.3 & 14.6 \\
\hline \multicolumn{4}{|l|}{ Percepción cambio de técnica } \\
\hline Nada afectado & 25.8 & 24.8 & 25.0 \\
\hline Ligeramente afectado & 57.7 & 56.5 & 56.7 \\
\hline Muy afectado & 12.7 & 16.7 & 16.0 \\
\hline $\mathrm{NS} / \mathrm{NR}$ & 3.8 & 2.0 & 2.3 \\
\hline \multicolumn{4}{|l|}{ Percepción cambio de rendimiento físico } \\
\hline Nada afectado & 10.9 & 18.9 & 17.5 \\
\hline Ligeramente afectado & 57.3 & 58.0 & 57.9 \\
\hline Muy afectado & 30.4 & 22.2 & 23.6 \\
\hline $\mathrm{NS} / \mathrm{NR}$ & 1.3 & 0.9 & 1.0 \\
\hline \multicolumn{4}{|l|}{$\begin{array}{l}\text { Inclusión de complemento/equipamiento } \\
\text { específico para la práctica }\end{array}$} \\
\hline Ya hacía uso & 8.5 & 13.3 & 12.5 \\
\hline Sí & 42.1 & 22.0 & 25.4 \\
\hline No & 33.4 & 50.5 & 47.6 \\
\hline No corresponde & 16.0 & 14.2 & 14.5 \\
\hline \multicolumn{4}{|l|}{ Tipo de complemento/equipamiento } \\
\hline Cinta de correr & 9.4 & 11.2 & 10.8 \\
\hline Elíptica & 2.9 & 4.2 & 3.9 \\
\hline Bicicleta estática & 12.8 & 18.4 & 17.2 \\
\hline Máquina de remo & 1.6 & 2.0 & 1.9 \\
\hline Rodillo bicicleta & 22.5 & 12.7 & 14.9 \\
\hline Pesos libres & 18.7 & 19.4 & 19.2 \\
\hline Cuerdas/elásticos/TRX/lastres & 21.1 & 20.2 & 20.4 \\
\hline Barras paralelas/elevación & 3.1 & 4.2 & 4.0 \\
\hline Aparatos de Home Gym & 6.1 & 6.9 & 6.7 \\
\hline Otros & 1.7 & 0.9 & 1.3 \\
\hline \multicolumn{4}{|l|}{ Adquisición de equipamientos } \\
\hline Sí & 14.5 & 9.2 & 10.1 \\
\hline No & 35.9 & 26.0 & 27.7 \\
\hline No corresponde & 49.6 & 64.8 & 62.2 \\
\hline \multicolumn{4}{|l|}{ Incorporación seguimiento redes sociales } \\
\hline Sí & 47.7 & 29.4 & 32.5 \\
\hline No & 52.3 & 70.4 & 67.5 \\
\hline \multicolumn{4}{|l|}{ Incorporación uso nueva App/Software } \\
\hline Sí & 25.7 & 14.0 & 16.0 \\
\hline No & 74.3 & 86.0 & 84.0 \\
\hline \multicolumn{4}{|l|}{ Incorporación entrenador/a deportivo } \\
\hline Sí & 33.3 & 12.0 & 15.6 \\
\hline No & 66.7 & 88.0 & 84.4 \\
\hline
\end{tabular}


En relación a la inclusión de complementos y/o equipamientos específicos para la práctica deportiva durante la fase de confinamiento, las personas encuestadas respondieron que en España si incorporaron nuevos elementos (42.1\%) mientras que en Portugal no (50.5\%). El equipamiento que más se incorporó fue el rodillo para bicicleta $(22.5 \%)$ y las cuerdas, elásticos, TRX, lastres (21.1\%) en el caso de España y las cuerdas, elásticos, TRX, lastres (20.2\%) y pesos libres (19.4\%) en Portugal. Aún así en ambos territorios la mayoría de las personas encuestados respondieron que no habían adquirido nuevos equipamientos (35.9\% en España y $26.0 \%$ en Portugal).

Finalmente, en relación al seguimiento del entrenamiento, la mayoría de las personas encuestados respondieron que no habían realizado un seguimiento por redes sociales (52.3\% en España y 70.4\% en Portugal), no habían utilizado nuevas aplicaciones o software (74.3\% España y $86.0 \%$ Portugal) y tampoco habían incorporado un entrenador/a (66.7\% España y $88.0 \%$ Portugal). Tabla 6.

Aún así, cabe subrayar que, aunque en ambos estados hubo una disminución de la frecuencia y duración de las sesiones, se perciben diferencias entre territorios. En cuanto al número de sesiones, en España aumentan los extremos y bajan las variables de 2-3 y de 4-6 sesiones; mientras que en Portugal suben todas las variables menos los de 4-6 que disminuyen. En relación al lugar de práctica, en España hay un cambio a práctica indoor (58.7\%) mientras que en Portugal hay una alternancia según las circunstancias (33.8\%).

En esta línea también, cabe tener en cuenta que en ambos territorios hubo la percepción de que hay una pérdida de su condición física (solo el 11\% en España y el 19\% en Portugal lo niegan) y de la capacidad técnica (solo el $25 \%$ dice que no) para las actividades deportivas. En España (42.1\%) incluyeron más complementos/equipamiento específico para la práctica que en Portugal (22\%), siendo los más incorporados el rodillo para bicicleta $(22.5 \%)$ y cuerdas/elásticos/TRX/ lastres $(21.1 \%)$ mientras que en Portugal incorporaron cuerdas/elásticos/TRX/lastres (20.2\%), pesos libres (19.4\%) y bicicletas estáticas (18.4\%).

Finalmente, destacar que en España la persona deportista está más expuesta al seguimiento en las redes sociales (47.7\%) respecto Portugal (29.4\%). Y que, la persona practicante española incorporó un entrenador/a guía en mayor medida (33.3\%) que su homólogo portugués (12\%).

\section{Práctica deportiva posterior al confinamiento}

En relación a la perspectiva para la fase post-COVID-19, la mayoría de las personas encuestadas respondieron que no iban a abandonar la práctica deportiva principal (89.9\% España y $94.3 \%$ Portugal) y tampoco iban a cambiar la forma de practicar la modalidad deportiva principal (55.1\% España y $49.8 \%$ Portugal). Sí que se mostraron ligeramente preocupados por el efecto de la COVID-19 en la práctica habitual y la futura participación en eventos deportivos (54\% en España y el 50\% en Portugal). En relación a la organización de eventos deportivos España y Portugal difirieron en su percepción. Así en España la mayoría creyó que iba a cambiar mucho (50.1\%), mientras que en Portugal la mayoría opinó que iba a cambiar algo (48.2\%). Finalmente, en relación al tiempo necesario para el retorno a la práctica, mientras que los datos obtenidos en ambos países apuntan a una media bastante similar (5.23 meses en el caso de España y 5.08 en el caso de Portugal).

En este sentido conviene destacar que los datos obtenidos muestran claramente un perfil de practicante consolidado y fiel a la práctica deportiva, así la mayoría (89.8\% en España y el 94.3\% en Portugal) no abandonó la modalidad deportiva principal. Tabla 7.

\begin{tabular}{|c|c|c|c|}
\hline Variables & $\begin{array}{c}\begin{array}{c}\text { España (\%) } \\
\mathrm{n}=451\end{array} \\
\end{array}$ & $\begin{array}{c}\begin{array}{c}\text { Portugal (\%) } \\
\mathrm{n}=2245\end{array} \\
\end{array}$ & $\begin{array}{c}\text { Total muestra (\%) } \\
\mathrm{N}=2696\end{array}$ \\
\hline \multicolumn{4}{|l|}{ Abandonó práctica modalidad principal } \\
\hline Sí & 1.1 & 0.3 & 0.5 \\
\hline No & 89.8 & 94.3 & 93.6 \\
\hline NS/NR & 9.1 & 5.4 & 5.9 \\
\hline \multicolumn{4}{|l|}{ Cambió en la forma de practicarla } \\
\hline Sí & 31.8 & 24.0 & 25.3 \\
\hline No & 55.1 & 49.8 & 50.8 \\
\hline $\mathrm{NS} / \mathrm{NR}$ & 13.1 & 26.2 & 23.9 \\
\hline \multicolumn{4}{|l|}{$\begin{array}{l}\text { Preocupación efecto de la COVID-19 en la } \\
\text { práctica deportiva habitual y futura } \\
\text { participación en eventos deportivos }\end{array}$} \\
\hline Ligeramente preocupado & 54.0 & 50.0 & 50.7 \\
\hline Muy preocupado & 19.6 & 38.8 & 35.6 \\
\hline Nada preocupado & 24.8 & 10.5 & 12.8 \\
\hline $\mathrm{NS} / \mathrm{NR}$ & 1.6 & 0.7 & 0.9 \\
\hline \multicolumn{4}{|l|}{ En relación a la organización de eventos } \\
\hline Creo que va a cambiar algo & 42.8 & 48.2 & 47.3 \\
\hline Creo que va a cambiar mucho & 50.1 & 43.6 & 44.7 \\
\hline No creo que cambie nada & 4.0 & 4.7 & 4.5 \\
\hline NS/NR & 3.1 & 3.5 & 3.5 \\
\hline $\begin{array}{l}\text { Tiempo necesario para retorno a la práctica } \\
\text { deportiva habitual (meses). Media (SD). } \\
\text { M [IOR]. }\end{array}$ & $\begin{array}{c}5.23(5.53) \\
4.00[2.00,6.00\end{array}$ & $\begin{array}{c}5.08(5.22) \\
3.00[2.00,6.00]\end{array}$ & $\begin{array}{c}5.10(5.27) \\
3.00[2.00,6.00]\end{array}$ \\
\hline
\end{tabular}

Se observa un perfil de practicante consolidado y fiel a la práctica deportiva, así la mayoría (89.8\% en España y el $94.3 \%$ en Portugal) no abandonó la modalidad deportiva principal.

\section{Discusión y conclusiones}

Este trabajo ha investigado el efecto que el estado de emergencia -en España- y el estado de calamidad -en Portugal- ha tenido en la práctica deportiva de las personas participantes en eventos deportivos de carrera a pie y ciclismo en España y Portugal, lo que lo convierte en un estudio pionero e implica una contribución novedosa, y hasta necesaria, en el ámbito sociológico de la práctica deportiva. Tanto es así que durante el proceso de pandemia internacional algunos investigadores han intentado conocer el impacto en los hábitos deportivos (Brooks, et al. 2020; Mutz \& Gerke, 2020; Schnitzer, et al., 2020)

A través del análisis de 2696 personas deportistas que participan en eventos deportivos de carrera a pie y ciclismo en España y Portugal, los resultados confirman que hubo una adaptación a la situación y que ésta fue diferente según el territorio.

Como apunte previo a la presentación de la conclusiones y discusión, cabe destacar la casi plena coincidencia, tanto en España como en Portugal, en el perfil genérico en todas las variables analizadas; el orden, por porcentaje, de las variables, e incluso los porcentajes de cada variable para las cinco dimensiones analizadas.

En primer lugar, en relación al perfil sociodemográfico, sobresale la concreción y como se asemeja el perfil de la persona practicante entre España y Portugal. La tipología de práctica principal, carrera a pie y bicicleta, y la fuente utilizada para contactar con el universo, plataformas de inscripción a eventos deportivos, puede haber condicionado la tipología de la muestra. Aún así, resulta interesante, desde 
un punto de vista de gestión y marketing, conocer el perfil sociodemográfico de las personas clientes de eventos deportivos de deportes de carrera a pie y ciclismo, incluso saber que el perfil es el mismo aún para territorios diferentes.

Interesante es conocer también que este perfil de población no se ha visto, o lo ha hecho mínimamente, afectado laboralmente por la COVID-19. Especial mención recibe la variable de género, así atendiendo los valores del eurobarómetro (European Union, 2018) las mujeres que dicen hacer deporte vigoroso son el 12\% en España y el 7\% en Portugal; mientras que los resultados del cuestionario apuntan al $21 \%$ en España y el $28 \%$ en Portugal. Valores más elevados que la media europea, indicador que puede indicar que España, y aún más Portugal, las políticas para introducir o facilitar la práctica deportiva de la mujer pueden estar funcionando.

En segundo lugar, en relación al perfil deportivo y práctica deportiva previa al confinamiento, al igual que la variable sociológica, destaca la coincidencia concreta de los datos y observar como son prácticamente calcos para ambos territorios. Se podría entender que el tipo de deporte condiciona el tipo de práctica, así en un mundo globalizado todas las personas practicantes de una determinada disciplina con objetivos parecidos, participar en eventos deportivos, siguen planificaciones y metodologías de entrenamiento parecidas.

Por otro lado, de la lectura de los datos y en relación al sistema deportivo de las modalidades analizadas, se puede entender que:

a) España tiene un sistema deportivo más maduro que Portugal. Así, atendiendo la variable antigüedad, España tiene mayor porcentaje de deportistas con una antigüedad superior a cinco años y Portugal mantiene márgenes más estrechos entre las franjas iniciales de práctica. Otro dato que podría corroborar esta hipótesis es que el número de practicantes de triatlón, siendo esta, según la literatura gris, una disciplina joven que está en proceso de introducción en España (Cejuela, 2015), es superior en España;

b) Portugal tiene un sistema deportivo más equilibrado y constante, así, las franjas de antigüedad están más equilibradas y no hay un salto tan grande como en España. Por contra en España, de los mismos datos, se puede interpretar que hubo un boom de práctica que no arrastró a generaciones posteriores, igual como ha sucedido con otras modalidades deportivas (Segui-Urbaneja \& Farías-Torbidoni, 2018);

c) España tiene un sistema deportivo más sustentado en el asociacionismo, así la práctica deportiva está más vinculada a entidades deportivas mientras que en Portugal se realiza en grupo de amigos; $y$

d) en España, las personas que practican deporte con un nivel intermedio, eliminando el nivel novel y veterano, están más involucradas, puesto que dedican más sesiones de entrenamiento.

En tercer lugar, en relación a la práctica deportiva durante el confinamiento, este es quizá donde la coyuntura y medidas políticas afectaron de manera más diferente a los dos estados. En España se aprobó el estado de confinamiento y se obligó a la reclusión total en domicilio lo que impidió toda práctica en el aire libre; por el contrario, en Portugal nunca se prohibió la ocupación del espacio público. Así se observa como en España claramente hubo un cambio a práctica indoor mientras que en Portugal hubo una alternancia según las circunstancias.

En cuarto lugar, en relación a la práctica deportiva posterior al confinamiento, destaca la coincidencia concreta de los datos y observar como son prácticamente calcos para ambos territorios. Se observa como los cambios producidos durante el período de confinamiento, entrenamiento y estilo de vida, llegaron a impactar las expectativas para el futuro y, aunque la gran mayoría de las personas practicantes reveló su intención de no abandonar el deporte practicado, fue contundente al afirmar que introduciría cambios en la forma de practicarlo.

Cabe tener en cuenta que las características y lógica interna de las modalidades deportivas analizadas, deportes individuales en el aire libre, permite entender que no se hayan producido, ni se producirán, grandes cambios en los hábitos deportivos de la población analizada, seguramente, cosa diferente ocurriría si se analizaran practicantes de deportes colectivos o deportes individuales de oposición en espacios deportivos cerrados.

En quinto lugar, en relación a la participación en eventos deportivos, parece también que existe una coincidencia con la idea de que habrá un retorno a la normalidad en las competiciones deportivas, aunque con la clara noción de que los organizadores introducirán cambios. Nada más lejos que la realidad, atendiendo al informe publicado por la Endurance Sports Coalition, apunta que sólo en los Estados Unidos más del $80 \%$ de los organizadores de eventos deportivos y el $90 \%$ de puestos de trabajo vinculados al sector, podrían desaparecer por la crisis económica que ha generado el coronavirus. Contabiliza que se han cancelado unos 50.000 eventos en todo el mundo y el $92 \%$ de los eventos de deportes de resistencia que tenían previsto disputarse en 2020 en Estados Unidos. Concluye que las modalidades deportivas de resistencia serán las más afectadas durante los meses de la pandemia mundial (ESP, 2020).

Por otro lado, al igual que apuntan atletas internacionales (Donnelly, 2020) los practicantes muestran una preocupación significativa con las condiciones de seguridad para la práctica de deportes y creen que las entidades organizadoras introducirán cambios muy significativos. Un elemento clave para entender esta percepción es entender que la COVID-19 no afecto ni social ni laboral ni económicamente a las personas encuestadas y por lo tanto estas, simplemente adaptaron su normalidad a la situación de confinamiento, pensando que sería un elemento pasajero y todo volvería a la normalidad.

Finalmente, y en la línea de las publicaciones más recientes en relación al efecto de la COVID-19 (Maugeri, et al. 2020; Moscoso-Sánchez, 2020), no cabe duda que la población, en un primer momento reacción adaptando sus hábitos a la situación de confinamiento, pero ya dejaba entrever que iba a implicar un cambio mucho más profundo. Sin duda alguna, el momento de confinamiento en que se respondieron los cuestionarios revelan un marco mental cortoplacista con el objetivo de, en un momento u otro, volver a la normalidad y en la que se entendía la situación como pasajera.

Transcurridos unos meses, parece que ya nada volverá a ser como era y que la irrupción de la COVID-19 supondrá el punto de inflexión hacia una sociedad diferente con hábitos 
deportivos diferentes que deberán ser atendidos con parámetros diferentes cuestionando todo lo establecido hasta ahora. El deporte, en tanto que es una actividad social (Moscoso, et al., 2020), se verá, como está ocurriendo, claramente afectado y deberá repensarse como es capaz de volver a generar una sinergia entre la actividad y la realidad social.

\section{Limitaciones del estudio y recomendaciones para futu- ros trabajos}

Son limitaciones de este estudio:

- la muestra: tanto el acceso, tres organizaciones especializadas en la inscripción de eventos deportivos, la participación entre los dos estados y el momento en que se realizaba el cuestionario, son condicionantes claros de los resultados obtenidos y de las conclusiones a las que se puede llegar.

Son perspectivas de futuro:

- realizar un nuevo cuestionario a la misma base de contactos con el objetivo de conocer como ha evolucionado su práctica deportiva y participación en eventos deportivos tras los meses posteriores al confinamiento.

- efectuar el seguimiento en el número de eventos deportivos realizados, adaptaciones de estos y evolución del número de inscritos.

Datos que permitirán entender si la evolución y el proceso de adaptación a la «nueva realidad» ha supuesto el punto de inflexión para la creación de un nuevo sistema deportivo.

\section{Agradecimientos}

Los autores agradecen a Lap2Go, Timing Lap e ITER5, que haya colaborado, de manera desinteresada, en el envío de la encuesta a las personas deportistas de sus respectivas bases de datos, al igual que agradecen a todas aquellas personas que han dedicado 5 minutos en responder el cuestionario y con ello que hayan colaborado con la sociedad científica y civil en conocer el efecto que ha tenido los estados de alarma y calamidad en los y las practicantes de carrera a pie y ciclismo españoles y portugueses.

También se agradece al soporte recibido por el Instituto Nacional de Educación Física de Cataluña (INEFC) de la Generalitat de Cataluña.

\section{Referencias}

Brooks, S.K., Webster, R.K., Smith, L, et al. (2020) The psychological impact of quarantine and how to reduce it: Rapid review of the evidence. The Lancet, 395, 912-920. DOI: 10.1016/S0140-6736(20)3046-8

Cejuela, R. \& Quiroga, J.E. (2015). Orígenes y antecedentes del triatlón. Sporttraining, 62, 31-43.

Consejo Superior de Deportes, CSD (2017). Encuesta de hábitos deportivos. Tomado el 2 de junio de 2020 en: http:/ /www.csd.gob.es/csd/sociedad/encuesta-de-habitos-deportivos/

Decreto de la Presidencia de la República nº14-A/2020, de 18 de marzo, en que se declara el estado de emergencia, con fundamento a una verificación de una situación de cala- midad pública.

Donnelly, P. (2020). We are the games: The COVID-19 pandemic and athletes' voices. Sociología del deporte, 1,35-40. DOI: 10.46661/socioldeporte. 5009.

European Union. (2018). Eurobarometer on sport and physical activity. Special Eurobarometer, 472. European Union.

Endurance Sports Coalition (2020). With Races Shut Down Due To COVID-19 Crisis, Running Industry Racing Against The Clock To Pay Bills Tomado el 18 de julio de 2020 en: https://www.endurancesportscoalition.org/.

Maugeri, G., Castrogiovanni, P., Battaglia, G., Pippi, R. \& D'Agata, V. (2020). The impact of physical activity on psychological health during Covid-19 pandemic in Italy. Hellyon, 6. DOI: 10.1016/j.heliyon.2020.e04315

Moscoso-Sánchez, D. (2020). El contexto del deporte en España durante la crisis sanitaria de la COVID-19. Sociología del deporte, 1, 15-19. DOI: 10.46661/ socioldeporte. 5000

Moscoso, D., Sánchez, R., Piedra, J. \& Vilanova, A. (2020). Pensar el deporte en tiempos de la COVID-19. El papel fundamental de la sociología. Sociología del Deporte, 1,9-11.

Mutz, M. \& Gerke, M. (2020). Sport and exercise in times of self-qurantine: How Germans changed their behavior at the beginning of the Covid-19 pandemic. International Review for the Sociology of Sport, 1-12. DOI: 10.1177/ 1012690220934335

Nogueira, R.M., Farías-Torbidoni, E.I. \& Pereira da Silva, C. (2021). Eventos de bicicleta de montaña en Portugal: características, distribución temporal y espacial e incidencia en la red nacional de áreas protegidas y clasificadas. RETOS, 39, 216-223.

Real Decreto 463/2020, de 14 de marzo, por el que se declara el estado de alarma para la gestión de la situación de crisis sanitaria ocasionada por el COVID-19.

Resolución Consejo de Ministros n³3-A/2020, 30 de abril de 2020. Diario de la República ${ }^{\circ} 85 / 2020,3^{\circ}$ suplemento, serie I de 2020-04-30, en la que se declara la situación de calamidad en el ámbito de la pandemia de la enfermedad COVID-19.

Segui-Urbaneja, J. \& Farias, E.I. (2018). El trail running (carreras de o por montaña) en España. Inicios, evolución y (actual) estado de la situación. RETOS, 33, 123-128.

Schnitzer, M., Schöttl, S.E., Kopp, M. \& Barth, M. (2020). COVID-19 stay-at-home order in Tyrol, Austria: sports and exercise behavior in change? Public Health, 185, 218-220. DOI: 10.1016/j.puhe.2020.06.042

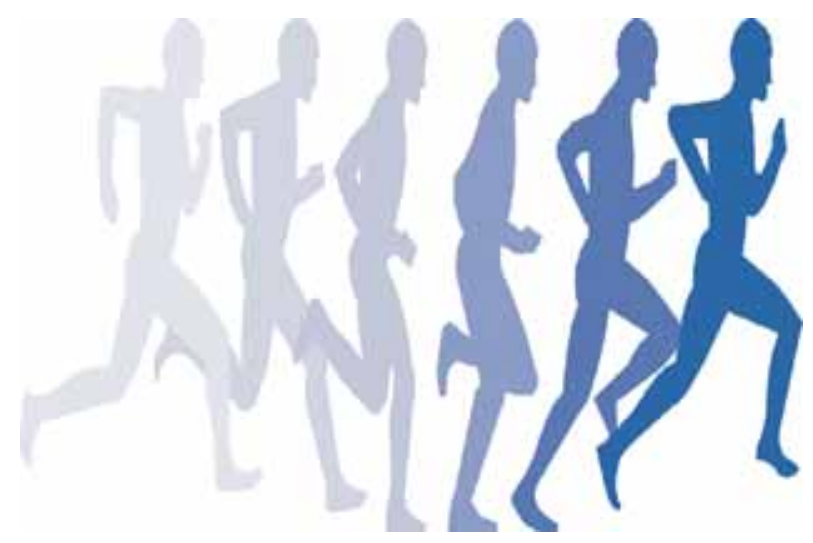

